\title{
Myiase intestinale humaine provoquée par des larves de Tipula paludosa, Meigen, 1930 (Diptera, Tipulidae) en Vendée par Jean GUEGAN
}

Chaire de Parasitologie et de Patologie exotique ( $\mathrm{P}^{\mathrm{r}} \mathrm{C}$. VermeIL)

Faculté de Médecine et de Parmacie, F 44 - Nantes

\section{Résumé}

Les auteurs décrivent une observation de myiase intestinale provoquée par la présence de larves de Diptères de la famille des Tipulidae appartenant à l'espèce Tipula paludosa Meigen 1830 chez un malade relevant du milieu rural vendéen.

\section{Summary}

A case of intestinal myiasis was described. It was due to the presence of larva of Tipulidae (Diptera) belonging to the species Tipula paludosa Meigen 1830 and found in a patient coming from the country in Vendée (France).

C'est à partir d'un prélèvement effectué à St Gilles-Croix-de-Vie en Vendée $\left(^{*}\right)$ dans les selles d'un malade atteint de dyspepsie hypersthénique que nous avons été amené à identifier des larves de Tipula paludosa, Meigen 1830.

(*) Nous remercions bien vivement le D' J. Alibert d'avoir bien voulu nous confier l'étude des larves récoltées. 
Cette catégorie de myiase, bien que signalée par quelques auteurs, ne semble pas avoir été jusqu'à présent bien définie (Leclercq : communication personnelle), aucune référence sur un cas précis n'est trouvable.
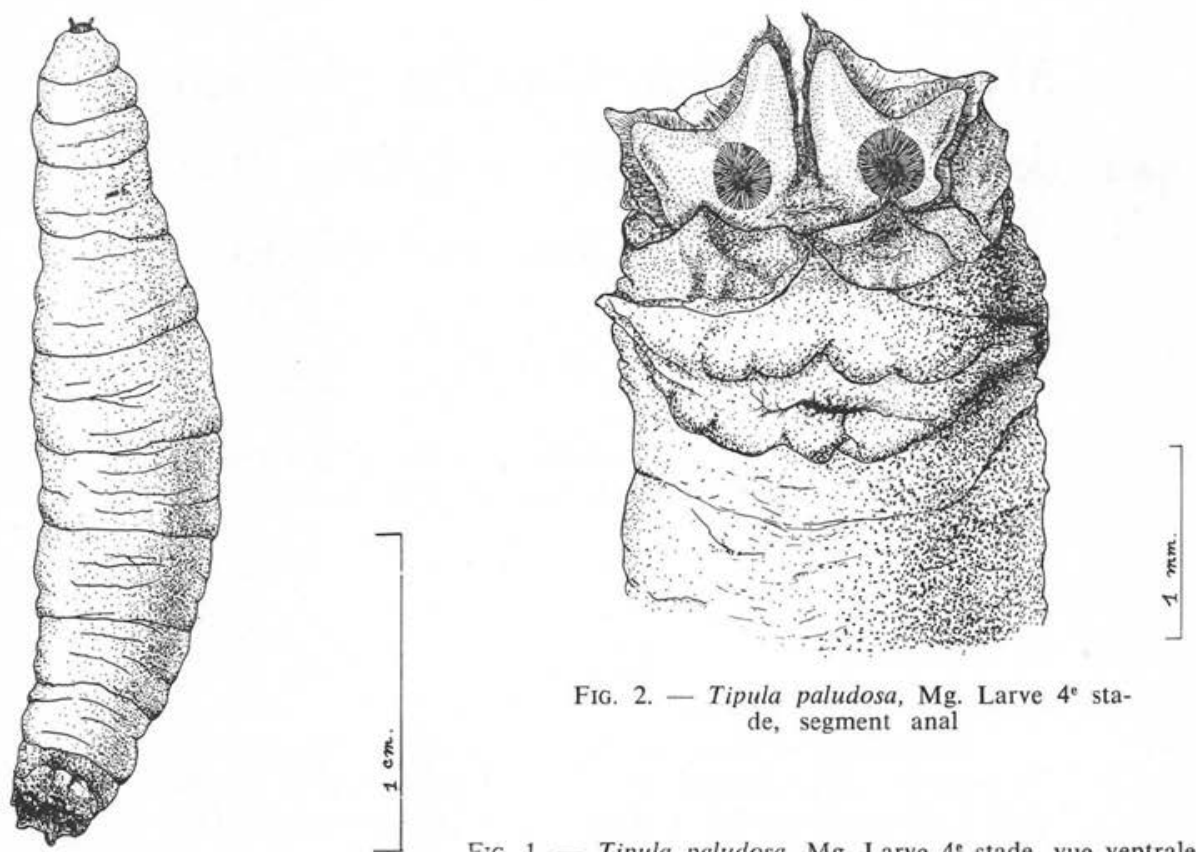

FIG. 2. - Tipula paludosa, Mg. Larve $4^{\mathrm{e}} \mathrm{sta}-$ de, segment anal

Fig. 1. - Tipula paludosa, Mg. Larve $4^{e}$ stade, vue ventrale

Pourtant, James (1), Smart (2), Mariani (3), Leclercq (4), citent les diptères de la famille des Tipulidae comme agents possibles de myiase intestinale.

La récente synthèse de Zumpt (5) ne les signale pas et n'implique parmi les nématocères, agents de myiases, que des représentants des deux families Anisopodidae et Psychodidae.

Donc, les œufs et les larves de diptères de la famille des Tipulidae peuvent être ingérés avec les aliments et être à l'origine d'un syndrome abdominal. Le malade qui fait l'objet de cette observation a été, pendant le mois de février 1969, victime d'un état dyspeptique hypersthénique avec diminution post-prandiale des douleurs et sensation de faim. Des accidents antérieurs de taeniasis conduisaient le malade à surveiller attentivement ses selles et de telle façon qu'un apport entomologique exogène pouvait être éliminé des produits d'exonération examinés, une dizaine de larves étaient ainsi récoltées vivantes. Les larves éliminées. les troubles digestifs disparaissaient complètement. 
La détermination générique de la larve qui nous est parvenue nous a été permise grâce aux deux ouvrages de Pierre (6) et de Bertrand (7), la précision d'espèce a nécessité l'utilisation des travaux de Brindle (8) (**) et de Théowald (9).

L'échantillon examiné était au quatrième stade et nous avons noté les caractéris. tiques suivantes:

- longueur : $25 \mathrm{~mm}$, largeur moyenne: $4 \mathrm{~mm}$, coloration brun foncé ;

- partie dorsale du prothorax sans marge saillante ;

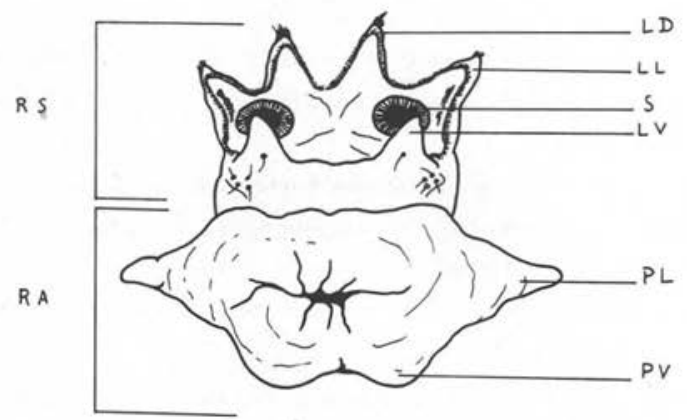

Fig. 3. - Tipula paludosa, Mg. Vue postérieure du segment anal de la larve

R.S. : région stigmatique, R.A. : région anale, L.D. : lobe dorsal, L.L.: lobe latéral, L.V.: lobe ventral, S. : disque stigmatique, P.L.: papille anale latérale, P.V : papille anale ventrale.

- lobes stigmatiques dorsaux bien séparés de longueur pratiquement égale aux lobes latéraux ; aplatis et assez larges, à pourtour semi-circulaire et pointus à l'extrémité ;

- absence de touffe de soies noires sur la partie dorsale du segment anal ;

- la paire latérale des papilles anales est plus allongée que les autres, la paire ventrale est arrondie ;

- les disques stigmatiques ne possèdent pas de taches brunes ;

- la bordure des lobes est mince et foncée ;

- une marque allongée brune se trouve en dehors de chaque stigmate.

Devant ces critères, nous devons considérer la différence de taille avec celle habituellement décrite, au maximum $44 \mathrm{~mm}$, comme une variation individuelle.

Dans les conditions normales, cette larve se développe dans les sols de pâturages et de marais, et même dans les sites plus strictement aquatiques. Cette espèce est, d'autre part, largement répandue.

(क⿳亠口冖丁) Nous remercions tout particulièrement le Dr A. Brindle du Muséum de Manchester qui a bien voulu confirmer notre détermination, ainsi que les $\mathrm{D}^{r s} \mathrm{G}$. Thompson et W. Bees.ey pour la documentation qu'ils ont bien voulu nous faire parvenir. 
L'observation accidentelle de larves de cette taille restées vivantes après un transit gastro-duodénal complet ne peut manquer de surprendre, si affamé qu'ait été le malade pour ne pas remarquer ce qu'il absorbait. Peut-être s'agissait-il de larves ingérées aux premiers stades et alors moins traumatisables par la mastication et qui auràient pu continuer à se développer quelque temps dans le milieu intestinal. la durée des symptômes nous le laisserait à penser.

\section{Bibliographie}

1. James (M.), 1947. - The flies that cause myiasis in man. U.S. Dept. Agric., Washington.

2. Smart (J.), 1956. - A handbook for the identification of insects of medical importance. British Museum, London.

3. Mariani (M.), 1956. - Compendio di entomologia medica. D.E.L.F., Palermo.

4. LeCLeCQ (M.), 1969. - Entomological parasitology, the relations between entomology and the medical Sciences. Pergamon, London.

5. ZUMPT (F.), 1965. - Myiasis in man and animals in the world, Butterworths, London.

6. Pierre (C.), 1924. - Diptères : Tipulidae. Lechevalier, Paris.

7. Bertrand (H.), 1954. - Les insectes aquatiques d'Europe. Lechevalier, Paris.

8. Brinde (A.), 1960. - The larvae and pupae of the British Tipulinae (Diptera: Tipulidae). Transac. Soc. British Entomology, 14, 63-114.

9. Theowald (B.), 1967. - Familie tipulidae (Diptera Nematocera). Larven und pupen Akademie, Berlin. 